\title{
NOUVELLE
}

\section{Exploiter les dépendances non-oncogéniques afin d'identifier de nouvelles cibles thérapeutiques dans les cancers pédiatriques}

Virginie Menez ${ }^{1}$, Marie-Anne Debily ${ }^{1,2}$

\author{
${ }^{1}$ Inserm U981, Molecular predictors and new targets \\ in oncology, Gustave Roussy, Université Paris-Saclay, \\ 94805 Villejuif, France. \\ ${ }^{2}$ Univ. Évry, Université Paris-Saclay, 91000 Évry, France. \\ marie-anne.debily@gustaveroussy.fr
}

Les cribles de létalité synthétique, un espoir pour la recherche en cancérologie

Depuis 2007, les techniques de séquençage de I'ADN à haut débit ont révolutionné la biologie et, en association avec les outils d' «ARN interférence » utilisés pour éteindre sélectivement l'expression d'un gène, ont permis une avancée majeure en génomique fonctionnelle en facilitant les criblages systématiques de «perte de fonction » dudit gène. La comparaison de cellules tumorales et de leur contrepartie non tumorale, dépourvue d'altération oncogénique, permet d'identifier rapidement les gènes dont l'inhibition permet de tuer uniquement les cellules cancéreuses. De cette façon, la présence d'altérations oncogéniques qui procurent un avantage aux cellules cancéreuses, mais qui sont souvent difficiles à cibler de manière systémique du fait de leur rôle majeur dans le fonctionnement cellulaire normal, est exploitée afin de les détruire sélectivement. Soixante-dixsept molécules candidates permettant d'induire la mort des cellules de cancer colorectal présentant des mutations de l'oncogène RAS ont ainsi été identifiées [11]. De même, dans une étude réalisée avec 58 lignées de cancer du poumon, BRM
(Brahma), un régulateur épigénétique essentiel à la prolifération des cellules cancéreuses mutées pour BRGI (Brahmarelated gene-1) est apparu comme une cible thérapeutique prometteuse [1].

Le gliome diffus de la ligne médiane, un cancer incurable

Le gliome diffus de la ligne médiane (diffuse midline glioma, DMG), la plus sévère des tumeurs cérébrales pédiatriques, affecte majoritairement des enfants âgés de 5 à 10 ans, qui décèdent dans un délai de deux ans après le diagnostic [2]. Ces tumeurs inopérables du fait de leur localisation dans le tronc cérébral et de leur caractère diffus sont particulièrement résistantes aux traitements, comme nous l'avons montré in vitro dans un crible chimique incluant 83 médicaments [3], et aucune chimiothérapie ne s'est avérée efficace dans les nombreux essais cliniques réalisés. La radiothérapie demeure le traitement de référence depuis plus de 50 ans, mais ses effets bénéfiques ne sont que transitoires.

En 2012, une mutation somatique du gène codant I'histone $\mathrm{H} 3(\mathrm{H} 3-\mathrm{K} 27 \mathrm{M})$, un acteur majeur du contrôle épigénétique de l'expression des gènes, a été identifiée comme l'évènement initiateur du développement de la tumeur du fait de sa fréquence très élevée ( $90 \%$ ) chez les patients $[4,5]$. Cette altération, jamais décrite dans d'autres cancers, entraîne la perte globale de la triméthylation du résidu lysine en position 27 de l'histone $\mathrm{H3}$ et une dérégulation majeure de l'expression de nombreux gènes, qui produit un contexte favorable à la transformation maligne des cellules. La substitution K27M est retrouvée majoritairement dans les gènes codant les histones H3.1 (15\% des cas) et $\mathrm{H3.3}$ (75\% des cas) [6]. Elle pourrait constituer une cible thérapeutique de choix dans cette maladie, mais ne peut être directement ciblée à l'heure actuelle, d'autant que la protéine mutée est dépourvue d'activité enzymatique propre. Ces cancers présentent peu d'anomalies génétiques additionnelles [10] $(\rightarrow)$ et aucune de celles trouvées de

$(\rightarrow)$ Voir la Nouvelle de P.Y. Dietrich et v. Dutoit, , page 319 de ce numéro manière récurrente ne peut être facilement ciblée dans un but thérapeutique. C'est le cas notamment des mutations de TP53 qui code la protéine p53, du fait du rôle central que joue cette protéine dans la réparation de l'ADN et dans le cycle cellulaire. Il est donc primordial d'améliorer notre connaissance du mécanisme tumo- 

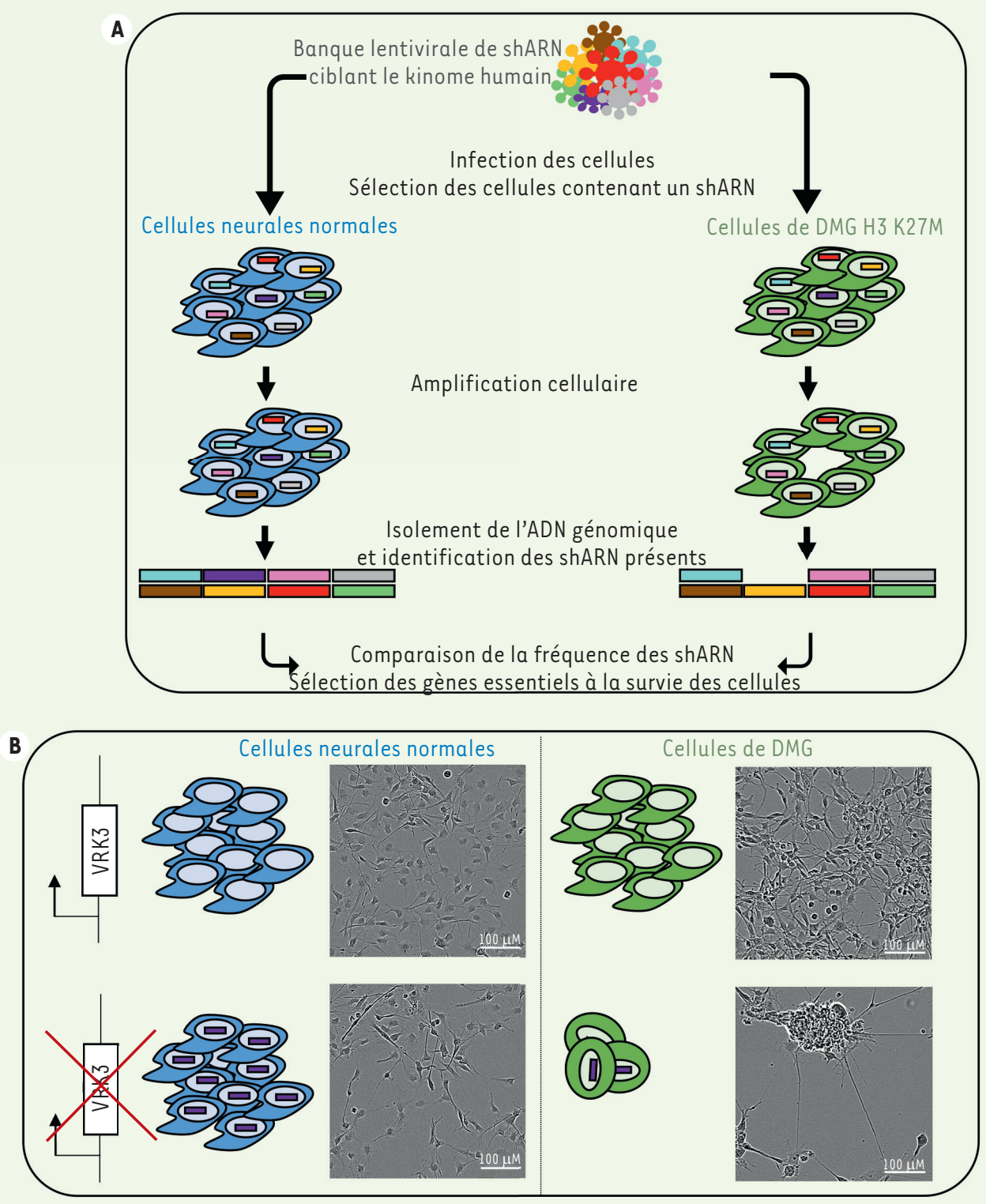

Figure 1. A. Stratégie du crible de létalité synthétique dans les DMG comportant la mutation H3-K27M. Les cellules neurales normales prises comme témoins et les cellules de DMG portant la mutation H3-K27M sont transduites avec une banque de vecteurs lentiviraux recombinants qui codent des shARN ciblant le kinome (i.e., l'ensemble des kinases) humain. Les cellules contenant un shARN sont sélectionnées, puis maintenues en culture. À 22 jours post-infection, I'ADN génomique des cellules est extrait, et les parties codant les shARN sont amplifiées par PCR et identifiées par séquençage à haut débit. Les shARN présentant une réduction significative de la fréquence de leur ADN codant parmi les cellules de DMG H3-K27M récoltées après 22 jours de culture post-infection (en violet), mais pas parmi les cellules témoins, sont sélectionnés. B. Effet de la répression de VRK3 sur les cellules de DMG H3-K27M. Cinq jours après introduction d'un shARN ciblant VRK3 (violet), on observe une diminution significative de la prolifération des cellules de DMG, associée à un changement de morphologie et un décollement des cellules, phénotype caractéristique de la mort cellulaire. Ce phénotype n'est en revanche pas observé avec les cellules neurales normales, ni avec les cellules de DMG ne présentant pas de répression de VRK3.

ral dans cette maladie et d'utiliser des approches sans a priori pour découvrir de nouvelles façons de traiter ce cancer encore incurable.

\section{Identification de vulnérabilités dans} les DMG

Nous avons entrepris la recherche, à grande échelle, de vulnérabilités des gliomes diffus de la ligne médiane en nous focalisant d'abord sur les 672 protéine kinases qui font fréquemment l'objet de thérapies ciblées inhi- 
bitrices [7]. Un crible a été réalisé à l'aide d'une banque de 7450 lentivirus recombinants, qui s'intègrent dans le génome des cellules hôtes et produisent différents ARN interférents (shorthairpin ARN, shARN), permettant ainsi d'éteindre de manière irréversible les gènes ciblés. Quatre modèles in vitro de DMG, développés dans notre laboratoire, utilisant des cellules souches de gliomes (glioma stem cells, GSC) dérivées de biopsies de patients ont été étudiés en parallèle : deux comportent une mutation de $\mathrm{H} 3.3$ et deux autres une mutation de $\mathrm{H} 3 . \mathrm{l}$, afin de prendre en compte l'hétérogénéité génétique de ces tumeurs. Deux modèles de cellules souches neurales (neural stem cells, NSC) normales cultivées dans des conditions identiques à celles des GSC ont été utilisés comme témoins négatifs. Dans ces modèles, les cellules sont transduites par les lentivirus codant les shARN de sorte qu'une cellule ne produise qu'un seul ARN interférent. La moitié des cellules sont collectées quarante heures après la transduction afin de vérifier, par séquençage à haut débit de l'ADN lentiviral intégré, la représentation de l'intégralité de la collection de shARN dans la population cellulaire. L'autre moitié des cellules transduites est maintenue en culture pendant 22 jours, et l'ensemble des shARN synthétisés par ces cellules est alors également caractérisé par séquençage de l'ADN lentiviral intégré. Les gènes essentiels à la survie des DMG sont identifiés grâce aux shARN qui les ciblent, car ceux-ci présentent une franche diminution de leur fréquence observée à 22 jours par rapport à 40 heures post-transduction dans les cellules cancéreuses, et dans une moindre mesure dans les cellules saines. En effet, cette déplétion du shARN à 22 jours témoigne d'un arrêt de la prolifération ou de la mort des cellules qui l'expriment, consécutivement à l'extinction du gène cible. La robustesse de la sélection des candidats est assurée pour partie par la redondance de la banque permettant de se focaliser sur les gènes pour lesquels plusieurs shARN présentent le même effet. De même, les gènes dont l'expression est vitale dans au moins trois des quatre modèles cellulaires de DMG ont été sélectionnés afin de s'assurer que les résultats étaient généralisables à l'ensemble des patients. Grâce à ce crible de létalité synthétique ${ }^{1}$, nous avons identifié 41 gènes nécessaires à la survie des cellules de DMG, parmi lesquels trois font actuellement l'objet d'une étude de l'effet de leur extinction dans des essais cliniques (Figure IA).

\section{VRK3, le talon d'achille des DMG}

Parmi les gènes candidats, VRK3, codant une sérine/thréonine kinase de la famille des vaccinia-related kinases (VRK), a retenu notre attention, notamment du fait des interactions de VRK3 avec plusieurs protéines impliquées dans l'assemblage de la chromatine, dont l'accessibilité est directement impactée par la mutation H3-K27M. Bien que ses fonctions demeurent assez mal connues, cette protéine a été impliquée dans la progression du cycle cellulaire (notamment dans le cancer du foie), dans la réparation de l'ADN, ou encore dans le développement neuronal $[8,9]$. II convient de noter qu'aucune mutation de VRK3 ou altération de son expression n'a jusqu'ici été identifiée dans les DMG, ce qui témoigne de l'efficacité d'une approche sans «a priori » pour dévoiler des gènes qu'une approche «classique » n'aurait jamais identifiés comme cibles thérapeutiques potentielles.

Nous avons validé l'impact majeur de l'inhibition de VRK3 sur la prolifération dans les quatre modèles de GSC, en uti-

\footnotetext{
${ }^{1}$ La létalité synthétique désigne le fait que deux évènements indépendants (mutations, dérégulation d'expression, etc.) qui, isolément, n'affectent pas la viabilité mais qui conduisent à la mort de la cellule lorsqu'ils se produisent simultanément. Dans notre étude sur des lignées cellulaires dérivées de DMG, le criblage a permis d'identifier les interactions létales entre la présence de la mutation K27M de I'histone $\mathrm{H} 3$ et l'extinction d'un second gène par l'expression d'un ARN interférent.
}

lisant 4 ARN interférents distincts qui ont entraîné chacun une répression de ce gène (Figure 1B). Nous avons montré, en utilisant une molécule imperméante ne pénétrant que dans les cellules mortes, que cet effet résultait d'une augmentation de la mortalité des cellules H3.3-K27M, alors que les cellules H3.1-K27M présentaient uniquement des altérations morphologiques associées à un arrêt du cycle cellulaire. Ce résultat suggère que le mécanisme de l'action anti-tumorale issue de la répression de VRK3 diffère dans les deux sous-groupes principaux de DMG.

\section{Perspectives}

Ce travail a révélé l'implication de nouvelles protéines, telles que VRK3, dans les DMG. La mise en place de cribles de létalité synthétique peut ainsi permettre de découvrir de nouvelles pistes thérapeutiques dans des cancers contre lesquels les traitements classiques sont très peu efficaces. II a été montré que la suppression totale de la protéine VRK3 par knock-out du gène correspondant dans des modèles murins entraîne des symptômes similaires à ceux présents dans l'autisme, mais n'altère pas la viabilité des souris [9]. Ce résultat suggère qu'un traitement systémique ciblant VRK3 chez les patients atteints de DMG pourrait ne pas avoir d'effet indésirable majeur sur les tissus sains. Des études in vivo devront confirmer l'effet bénéfique de l'extinction de $V R K 3$ dans les modèles de xénogreffe tumorale que nous avons développés au laboratoire. De plus, aucun inhibiteur de la protéine VRK3 n'étant disponible à I'heure actuelle, il est logique de s'intéresser aux ligands de cette protéine et aux conséquences moléculaires de la répression de VRK3 dans ces tumeurs afin d'identifier de nouvelles cibles thérapeutiques potentielles, dont la manipulation mimerait l'effet anti-tumoral de la répression de VRK3. $\diamond$

Identifying essential non-oncogenic addictions to define new therapeutic targets in pediatric cancer 


\section{LIENS D'INTÉRÊT}

Les auteures déclarent n'avoir aucun lien d'intérêt concernant les données publiées dans cet article.

\section{RÉFÉRENCES}

1. Hoffman GR, Rahal R, Buxton F, et al. Functional epigenetics approach identifies BRM/SMARCA2 as a critical synthetic lethal target in BRGl-deficient cancers. Proc Natl Acad Sci USA 2014 ; 111 : 3128-33.

2. Puget $S$, Beccaria K, Blauwblomme $T$, et al. Biopsy in a series of 130 pediatric diffuse intrinsic Pontine gliomas. Childs Nerv Syst $2015 ; 31: 1773-80$.

3. Grasso CS, Tang Y, Truffaux N, et al. Functionally defined therapeutic targets in diffuse intrinsic pontine glioma. Nat Med $2015 ; 21: 555-9$.
4. Schwartzentruber J, Korshunov A, Liu XY, et al. Driver mutations in histone $\mathrm{H} 3.3$ and chromatin remodelling genes in paediatric glioblastoma. Nature 2012 ; 482 226-31.

5. Wu G, Broniscer A, McEachron TA, et al. Somatic histone $\mathrm{H} 3$ alterations in pediatric diffuse intrinsic pontine gliomas and non-brainstem glioblastomas. Nat Genet 2012 ; 44 : 251-3.

6. Castel D, Philippe C, Calmon R, et al. Histone H3F3A and HIST1H3B K27M mutations define two subgroups of diffuse intrinsic pontine gliomas with different prognosis and phenotypes. Acta Neuropathol $2015 ; 130$ : 815-27.

7. Silva-Evangelista $C$, Barret $\varepsilon$, Ménez $V$, et al. $A$ kinome-wide shRNA screen uncovers vaccinia-related kinase 3 (VRK3) as an essential gene for diffuse intrinsic pontine glioma survival. Oncogene 2019 ; 38 : 6479-90.
8. Lee N, Kim DK, Han SH, et al. Comparative Interactomes of VRKI and VRK3 with their distinct roles in the cell cycle of liver cancer. Mol Cells 2017 ; $40: 621-31$.

9. Kang M-S, Choi TY, Ryu HG, et al. Autism-like behavior caused by deletion of vaccinia-related kinase 3 is improved by TrkB stimulation. J Exp Med 2017 ; 214 : 2947-66.

10. Dietrich Py, Dutoit V. Stratégies de vaccination thérapeutique dans le glioblastome. Med Sci (Paris) $2020 ; 36: 319-22$.

11. Luo J, Emanuele MJ, Li D, et al. A genome-wide RNAi screen identifies multiple synthetic lethal interactions with the Ras oncogene. Cell 2009 ; 137 : 835-48.

NOUVELLE 